\title{
Evolución de los conceptos y paradigmas que orientan la gestión ambiental ¿cuáles son sus limitaciones desde lo glocal?
}

\author{
Juan Pablo Martínez Idrobo* \\ Apolinar Figueroa Casas**
}

Recibido: 12/09/2013 • Aceptado: 11/10/2013

\begin{abstract}
Resumen
El presente artículo aborda la evolución cronológica de diferentes paradigmas y conceptos relacionados con la gestión ambiental (GA). Para ello, hace énfasis en aquellos que han incidido en la dirección de la GA en el país, e identifica el concepto de desarrollo asociado, así como los planteamientos y dimensiones considerados para establecer las relaciones existentes entre los mismos. Si bien la gestión ambiental es una de las funciones públicas compartidas solidariamente con la sociedad, su implementación ha sido unidireccional, lo que ha promovido modelos homogeneizadores que limitan la interacción con las comunidades y las relaciones interinstitucionales e interdisciplinares. Si se observa esta situación desde una lógica glocal en diferentes ecosistemas y regiones, es evidente el predominio del enfoque de crecimiento económico con una escasa interacción entre saberes y conocimiento, situación que limita la construcción de alternativas de manejo sostenible y desencadena acciones ambientales desarticuladas.
\end{abstract}

Palabras clave: gestión, ecosistemas, ambiente, paradigma.

Biólogo, candidato a Doctor en Ciencias Ambientales, investigador asociado Grupo de Estudios Ambientales, Universidad del Cauca. Museo de Historia Natural - Carrera 2 N. 1A-25, Of.206 Popayán (Col), Tel. 8209800 Ext.2645.jpmartinez@unicauca.edu.co

** Biólogo, doctor en Ciencias Biológicas, director Grupo de Estudios Ambientales, profesor Universidad del Cauca. Museo de Historia Natural - Carrera 2 N.1A-25, Of.206 Popayán (Col), Tel. 8209800 Ext. 2645. apolinar@ unicauca.edu.co 


\title{
Evolution of the concepts and paradigms that guide the environmental management; what are their limitations in the glocal context?
}

\begin{abstract}
In this article was made an analysis of the chronological evolution of different paradigms and concepts related to the environmental management (EM). the review highlights those that have impacts on the guidance of the EM in the country and the concept of associated development, as well as the considered approaches and dimensions for establishing the relations between them. Although the environmental management is one of the public functions shared with the society, the implemented model of EM has been unidirectional and this limited the interinstitutional interaction with the communities and the interdisciplinary relations. If this situation have a glocal-logic point of view in different ecosystems and regions, the predominance of the economic growth approach is evident with a low interaction between know-how and knowledge, that circumstance restrict the construction of sustainable management alternatives and generate environmental actions isolated. Key words: management, ecosystems, environment, paradigm. Key words: management, ecosystems, environment, paradigm.
\end{abstract}




\section{INTRODUCCIÓN}

Las relaciones entre los seres humanos y su entorno han estado mediadas por la necesidad de satisfacer sus requerimientos como especie y constructo colectivo; por ello, la humanidad ha generado una multiplicidad de interacciones socioecológicas, ya que las concepciones y mecanismos empleados para relacionarse con la naturaleza han sido tan diversos como la oferta de ambientes y grupos socioculturales que existen en la biosfera.

Debe reconocerse entonces, que la oferta natural ha sido el sustrato del progreso económico y el bienestar social, proporcionando los recursos requeridos para mejorar la calidad de vida de las poblaciones, entendiendo esta última como la satisfacción de las necesidades humanas, de los deseos y aspiraciones de las personas, alcanzables mediante satisfactores materiales, alternativos e inmateriales [1-3].

Si bien la naturaleza se incorpora diferencialmente en los sectores económicos es innegable su aporte en el mantenimiento y dinamización de los mismos. Producto de esta incorporación, principalmente con fines económicos materiales, los resultados tangibles evidencian una situación desequilibrada que ha generado impactos negativos, en múltiples escalas y ha propiciado el detrimento de la base natural favoreciendo la concentración del capital económico y la inequidad [4, 5], situación que no debe entenderse como "desarrollo".

\section{GESTIÓN AMBIENTAL, DESARROLLO Y GLOBALIDAD, EL PUNTO DE PARTIDA}

Según lo expuesto anteriormente, puede plantearse que el desarrollo se enfoca en el mejoramiento de la calidad de vida de las personas, sin que ello priorice el crecimiento económico, ya que el término desarrollo apunta claramente a la idea de cambio gradual y direccional, en el sentido de mejoramiento [6]. Por otra parte, el desarrollo no significa necesariamente crecimiento cuantitativo, ya que se refiere esencialmente al despliegue de potencialidades en un entorno de complejidad creciente. En conclusión, puede entenderse el desarrollo como la búsqueda del bienestar colectivo e individual, a través del aprovechamiento de los bienes y servicios que oferta la naturaleza, en múltiples escalas espaciotemporales de los sistemas co-evolutivos.

Tradicionalmente la gestión ambiental se ha enfocado principalmente en la administración de los recursos naturales contenidos en unidades espaciales territoriales o ecológicas [7-17], asumiéndolos como un capital natural aprovechable perteneciente a una organización determinada, pública o privada, bajo un enfoque predominante de sostenibilidad débil. Si bien se procura la integración de los sistemas natural, social y económico bajo una lógica lineal, esta relación intenta mantener o incrementar el capital total en una temporalidad limitada, situación que favorece el remplazo de los capitales interactuantes como el natural [18]. 
La lógica expuesta en el párrafo anterior se sustenta en el relacionamiento unidireccional desde los niveles gubernamentales e institucionales macro y meso a través de una aproximación Top-Down, situación que no incorpora los aportes desde el territorio hacia lo regional y nacional Bottom-Up. En este sentido el concepto de Glocalización reconoce que las localidades son casi siempre el resultado de un proceso histórico endógeno amplio y, a la vez, producto de interacciones globales exógenas [19, 20]; esta noción tiene en cuenta que la condición de globalidad genera una interpretación multiescalar del mundo, que es simultáneamente espacial y temporal, geográfico e histórico.

Considerando lo anterior y para orientar la exposición, a partir de este punto, se trabajarán los paradigmas o conceptos fundamentales y el planteamiento de desarrollo asociado a la gestión ambiental, dado que la relación entre los conceptos de gestión ambiental y desarrollo estará mediada por el entendido de coexistir en una lógica causal realimentada de mecanismo-finalidad que se transforma y obliga al ajuste mutuo.

\section{EL REPORTE DE COLBY AL BANCO MUNDIAL}

A continuación se presentaran doce paradigmas de la gestión ambiental extraídos de la literatura, empleando como punto de partida el reporte de Colby [21] al Banco Mundial donde se identifican los cinco primeros; en este sentido es interesante observar la evolución de los paradigmas en el tiempo y sus relaciones; por ello se analizarán cronológicamente.

El primer paradigma es la a) Economía de Frontera, que posicionaba el uso de la naturaleza como herramienta para el beneficio humano, y relega el ambiente a una condición de objeto manipulable a conveniencia sobre el cual se pueden aplicar las estrategias que se requirieren para fomentar el acopio y concentración material como ideal de calidad de vida. Este imaginario, aún vigente, alcanzó su culmen en la década de los setenta y sus máximos exponentes son los países desarrollados.

La gestión se caracterizó por ser antropocéntrica buscando el dominio científicotecnológico sobre la naturaleza, gestión cuya orientación estaba dada para atender las demandas de los sectores industriales y productivos, considerando que la oferta natural es ilimitada y que el ambiente tiene la capacidad permanente de asimilar los residuos de cualquier actividad antrópica. Se buscaba entonces el crecimiento económico material sin considerar los efectos sobre el entorno y el colectivo social, situación que precipitó la revaluación de este paradigma por la situación global de deterioro y el auge de los movimiento sociales que reclamaban, al nivel político, conciencia y corresponsabilidad ambiental.

En esta dinámica sociopolítica emerge el segundo paradigma, denominado b) Ecología Profunda como antípoda al anterior, reaccionando contra los efectos del modelo económico dominante que no consideraba aspectos éticos, sociales ni morales. 
En esta concepción es el ambiente el que subordina al ser humano y en esa condición propende por una gestión orientada a la mínima intervención sobre la naturaleza, procurando la reducción de la población, un retorno al uso de tecnologías y mecanismos de gestión propios de las comunidades ancestrales, entre otros. Sin embargo, por las implicaciones y los cambios institucionales, jurídicos, productivos que perseguía y su impacto radical en el modo de vida de las comunidades occidentalizadas su viabilidad fue mínima. Debe reconocerse que a pesar de ser una reflexión que no trascendió del propósito filantrópico, académicamente ciertos preceptos se han incorporado en las conceptualizaciones recientes del desarrollo.

Ante los evidentes problemas causados por la contaminación y la degradación ecosistémica, producto de la dinámica industrial, muchos Estados del Primer Mundo reaccionaron y crearon una institucionalidad que se ocupara de los asuntos medioambientales centrándose en el control del impacto de las actividades humanas sobre la naturaleza. Es así como en la década del sesenta, aparece la c) Protección Ambiental como el paradigma que permitió el desarrollo de instrumentos normativos, legales y técnicos [22] que valoraran la relación costo/beneficio de la contaminación, favoreciendo la creación de organismos e instancias estatales responsables de este nuevo sector. Sin embargo, uno de los principales problemas que tuvo este enfoque en el proceso de gestión fue la falta de conocimiento e información para establecer los niveles ecológicos que garantizaban la resiliencia de los sistemas naturales, dándole prioridad a la viabilidad económica de las empresas sobre el impacto ambiental que pudiesen estar ocasionando en el entorno.

De forma simultánea, en la Conferencia de las Naciones Unidas sobre Ambiente Humano (1972), se introduce el d) Ecodesarrollo en procura de cambios sustanciales en la forma de concebir el desarrollo [23]. Este planteamiento propende por un modelo económico acoplado termodinámicamente con los ecosistemas, que considere los ciclos de realimentación, reivindique las necesidades de los países del Tercer Mundo, intente desplazar el euro-centrismo presente en las teorías economicistas del desarrollo, y se preocupe por aspectos culturales y de equidad social que favorezcan la convergencia en el ecocentrismo, como indican algunos autores [24, 25].

Este planteamiento se fundamenta en tres aspectos: la libertad y autonomía para decidir, un desarrollo con equidad y prudencia ecológica, y un crecimiento cualitativo que permitiese armonizar las diferentes esferas del desarrollo. Esta propuesta debería conducir a un nuevo modelo económico que incorporara el principio de precaución y la incertidumbre ecológica en la planificación y gestión ambiental del sistema.

Hacia finales de los ochenta, se consolida la e) Administración de Recursos como un nuevo paradigma en consonancia con el propósito de las Naciones Unidas por globalizar la preocupación existente sobre los activos naturales y su incidencia en el 
desarrollo de los países; como objetivo principal se trazó la inclusión de los diferentes recursos naturales en los balances nacionales. Esta conceptualización promovió el desarrollo de métodos más precisos para el monitoreo de la oferta natural y la contaminación: las estrategias de gestión se relacionaron con el uso de tecnologías limpias, la conservación, la eficiencia energética, el principio del contaminador pagador y la salud del ecosistema, buscando incorporar productos diferenciados desde lo ambiental en segmentos específicos del mercado.

En este periodo, las Naciones Unidas presentan el f) Desarrollo Sostenible (DS) a través del Informe Bruntland [26], y lo definen como "el desarrollo que satisface las necesidades del presente sin comprometer la habilidad de las generaciones futuras de satisfacer sus propias necesidades"; este enunciado se refirma más adelante en la Declaración de Río de Janeiro (1992), la cual desglosa los 3 Objetivos del DS, i) Ecológicos que hacen referencia a la conservación del estado natural de los ecosistemas para garantizar la supervivencia en el tiempo, los ii) Económicos orientados a promover la productividad económica implementando tecnologías para el manejo sostenible de los recursos y los iii) Sociales enfocados en la distribución equitativa de los beneficios y costos del desarrollo entre la población.

Según Gallopín [27], el desarrollo sostenible implica un proceso de cambio mejoramiento que se puede mantener en el tiempo; planteamiento que requiere diferenciar la sostenibilidad como un principio funcional aplicable a determinados sistemas, y que hace referencia a la capacidad de mantenimiento en el tiempo de una situación o condición, mientras que el concepto de desarrollo implica específicamente un cambio de situación o condición, no su mantenimiento, y en este sentido los objetivos que persiga el desarrollo determinarán si este es sostenible o no.

Como señala Rockström [28], la confluencia de las aspiraciones insatisfechas del bienestar humano, incluyendo el desarrollo económico, y los límites de los ecosistemas que brindan soporte, obliga a definir un nuevo marco para el desarrollo sostenible que permita el desarrollo humano dentro de los límites de los sistemas que sustentan la vida en la Tierra. Por esto, es factible plantear que el desarrollo, para ser sostenible, no puede privilegiar un solo propósito o darle mayor énfasis [29], como ha ocurrido con lo económico y los niveles de consumo material, sino que deberá incluir de forma balanceada objetivos ecológicos, sociales, institucionales, inmateriales, entre otros, según determinadas escalas de valores y en contextos que varían en el tiempo, características que hacen de este, un proceso abierto que se alimenta progresivamente.

\section{MÁS ALLÁ DEL DESARROLLO SOSTENIBLE}

A finales de los ochenta y principios de los noventa, autores como Escobar [30] introducen la idea del $g$ ) Posdesarrollo, adjetivado también como sostenible, como una 
corriente crítica e insurgente al discurso desarrollista de las naciones industrializadas y su incidencia en países del Tercer Mundo. El posdesarrollo considera la posibilidad de generar nuevas cosmovisiones que no estén determinadas por la idea predominante del desarrollo favoreciendo la emergencia o reivindicación de prácticas, conocimientos, saberes y una economía política de la verdad que hagan visibles a las comunidades objeto del desarrollo y las transforma en sujetos y agentes del mismo. Según Escobar y otros autores [31-33], el posdesarrollo implicará en la práctica hacer énfasis en los procesos más que en los resultados, fomentando la reflexión inclusiva de los involucrados para configurar las metas y enfoques, y facilitar la comprensión en la toma de decisiones.

Estos debates motivaron el aporte de la Sociedad Ecológica Americana (SEA), que a mediados de los noventa presentó la i) Gestión de los Ecosistemas [34], reiterando la necesidad de una institucionalidad sólida que establezca políticas, normas y protocolos que se basen en la comprensión científica sustentada de las interacciones y procesos ecológicos necesarios para mantener la estructura, función y dinámicas de los ecosistemas para alcanzar metas precisas del desarrollo. Este planteamiento reivindicó la necesidad de una gestión multisectorial e interdisciplinaria soportada en el conocimiento ajustable a nuevos retos mediante el monitoreo e investigación.

En este sentido, la SEA criticaba los métodos de gestión observados en la realidad por su enfoque hacia la rentabilidad económica cortoplacista no sostenible, y convocaba nuevamente a la comprensión de la complejidad e interconectividad propia del carácter dinámico de los ecosistemas, donde los seres humanos son uno de los componentes $\mathrm{y}$, por tanto, es perentorio el compromiso con la adaptabilidad y la responsabilidad en diferentes contextos y escalas.

Como respuesta a la presión normativa que se había iniciado en materia ambiental en muchos de los países desarrollados a finales de la década de los setenta, y de forma articulada a la dinámica global de la mejora continua promovida por la Organización Internacional de Normalización (ISO), aparece en los noventa la $j$ ) Gestión Ambiental Integrada [35], fundamento de los sistemas de gestión ambiental para la industria y la empresa, con un énfasis hacia el manejo de los recursos, insumos, e impactos, desechos-subproductos, generados en distinta actividades de transformación [36].

Esta iniciativa alentó a la industria para que desarrollara sistemas de gestión ambiental que atendieran el proceso productivo aplicando conceptos de mejora con la finalidad de disminuir sus costes y aumentar la productividad, reducir, al tiempo, la contaminación y dar cumplimiento a las normativas existentes; esto se acompañó del desarrollo de la conciencia ambiental en los empresarios y les permitió diferenciarse mediante la implementación de estándares internacionales como las normas ISO 14001 [37]. 


\section{INCORPORANDO LA ADAPTACIÓN, LA INNOVACIÓN Y EL DECRECIMIENTO}

Para este periodo, y dada la necesidad de contar con una aproximación que incorporase elementos de anticipación y participación, aparece en el contexto científico la $k$ ) Gestión Adaptativa y Cogestión. Según Hole [38] se trata de un proceso interactivo ajustable para tomar decisiones adecuadas procurando reducir la incertidumbre a través del monitoreo y el aprendizaje. En este sentido, es necesaria la revisión y actualización periódica de los objetivos de la gestión (desarrollo), para incorporar herramientas de modelado y analizar las causas de los cambios observados en el sistema como base predictiva.

De igual forma se requiere una gama de opciones de gestión cuya implementación sea monitoreada y evaluada para determinar las mejores opciones, tanto para lo observado como para los escenarios proyectados, para desarrollar una estructura colaborativa que permita la participación de los tomadores de decisiones e interesados, e incorporar lecciones aprendidas [39, 40]. La cogestión adaptativa es un resultado de largo plazo de las experiencias de gestión colaborativas en las cuales se fortalece el aprendizaje y la articulación interinstitucional en varias escalas de gobernanza $[41,42]$.

De forma simultánea, a finales de los noventa, y producto de la naciente sexta ola de innovación global aparece la $l$ ) Ecoinnovación, entendida como la innovación que mejora el rendimiento ambiental, en consonancia con la resiliencia de los socioecosistemas, para buscar la reducción de los impactos adversos del desarrollo en el medio $[43,44]$ y la aplicación del conocimiento con el fin de obtener servicios y productos de tercera generación.

Como la innovación es altamente dependiente del contexto, al focalizarla en lo ambiental las particularidades que hacen único un proceso requieren de la integración de múltiples escalas, niveles de conocimiento y actores; para llegar a la Ecoinnovación deberá apostarse por una estrategia concreta, ya que aquello que es innovador para un grupo particular, en un determinado espacio y momento, puede ser totalmente fútil para otro contexto socionatural [45-47]. Sin embargo, este planteamiento aún está en construcción, ya que pretende trascender el enfoque positivista tecnológico que supedita la naturaleza a una condición de proveedor de materia y energía, sin perder de vista las interacciones entre economía, conocimiento, tecnologías y ambiente.

Finalmente se referirá el $\mathrm{m}$ ) Decrecimiento Sostenible, definido como un proceso colectivo deliberativo dirigido a la reducción equitativa de la capacidad general de producir y consumir, y al papel de los mercados y los intercambios comerciales como un principio central para la organización de la vida humana [48]; también ha sido nutrido por debates sociales como la Justicia Ambiental y el Ecologismo de los Pobres en favor de una economía sostenible [49].

Revista Ingenierías Universidad de Medellín 
Según Sekulova y colaboradores [50], la propuesta del decrecimiento debe integrar dos enfoques: el primero es la reducción de la complejidad social y económica en términos de la relocalización de la producción, reducción de intermediarios, disminución de los bienes consumidos por hogar, entre otras. El segundo hace referencia a su gestión e influencia en el contexto social, abordando las macro-medidas de adaptación que respondan a la complejidad existente, de forma tal que faciliten herramientas jurídicas compartidas, difusión global y el establecimiento de los límites no transables en la extracción de los recursos naturales, para fortalecer los estándares sociales y ecológicos.

\section{IDENTIFICANDO LAS LIMITACIONES Y SUS OPORTUNIDADES}

Para establecer los elementos diferenciales entre los paradigmas y conceptos desglosados anteriormente, a continuación se presentan en la tabla 1, los aspectos más relevantes identificados en cada uno de ellos, relacionando el concepto de desarrollo, con el planteamiento central y las dimensiones consideradas en cada uno.

Tabla 1. Descripción sintética de los paradigmas de gestión ambiental.

\begin{tabular}{|c|c|c|c|}
\hline Paradigma & Concepto de desarrollo & Planteamiento & Dimensiones \\
\hline $\begin{array}{l}\text { Economía de } \\
\text { Frontera } \\
\text { S. XVIII (RI) - } \\
1960(?)\end{array}$ & $\begin{array}{l}\text { Crecimiento económico } \\
\text { material asociado al capital } \\
\text { natural, enfoque de merca- } \\
\text { dos. Acopio y concentración } \\
\text { material como ideal de ca- } \\
\text { lidad de vida. La economía } \\
\text { y la sociedad subordinan la } \\
\text { naturaleza. }\end{array}$ & $\begin{array}{l}\text { Antropocéntrico, Enfoque po- } \\
\text { sitivista de optimismo tecno- } \\
\text { lógico, prima el crecimiento, } \\
\text { la naturaleza es manipulable y } \\
\text { reemplazable. }\end{array}$ & $\begin{array}{l}\text { Económico, Institucional } \\
\text { (Conocimiento y tecnolo- } \\
\text { gía), Biofísica (Materiales } \\
\text { y energía). }\end{array}$ \\
\hline $\begin{array}{l}\text { Ecología } \\
\text { Profunda } \\
1960 \text { (?) }\end{array}$ & $\begin{array}{l}\text { El ambiente subordina al ser } \\
\text { humano, gestión orientada a } \\
\text { la mínima intervención sobre } \\
\text { la naturaleza, sin crecimien- } \\
\text { to cuantitativo (antípoda al } \\
\text { Desarrollo). }\end{array}$ & $\begin{array}{l}\text { Ecocentrista, procura la reduc- } \\
\text { ción de la población, retorno al } \\
\text { uso de tecnologías y mecanismos } \\
\text { de gestión propios de las comu- } \\
\text { nidades ancestrales, entre otros. }\end{array}$ & $\begin{array}{l}\text { Biofísico (Ecológico), } \\
\text { Social, Institucional } \\
\text { Económico. }\end{array}$ \\
\hline $\begin{array}{l}\text { Protección } \\
\text { Ambiental } \\
1970\end{array}$ & $\begin{array}{l}\text { Crecimiento económico } \\
\text { asociado al capital natural, } \\
\text { preocupación por el sector } \\
\text { primario y secundario, enfo- } \\
\text { que de mercados. }\end{array}$ & $\begin{array}{l}\text { Normativo, generar instrumen- } \\
\text { tos jurídicos y técnicos que valo- } \\
\text { raran la relación costo/beneficio } \\
\text { de la contaminación. Creación } \\
\text { de instancias gubernamentales } \\
\text { responsables del ambiente. }\end{array}$ & $\begin{array}{l}\text { Institucional (conocimien- } \\
\text { to), Biofísico (Ecológico), } \\
\text { Económico (Industria). }\end{array}$ \\
\hline $\begin{array}{l}\text { Ecodesarrollo } \\
1970-1980\end{array}$ & $\begin{array}{l}\text { Modelo económico acoplado } \\
\text { termodinámicamente con los } \\
\text { ecosistemas considerando los } \\
\text { ciclos de realimentación (Pre- } \\
\text { cursor del DS) }\end{array}$ & $\begin{array}{l}\text { Estructuralista, armonización de } \\
\text { tres aspectos, libertad y autono- } \\
\text { mía para decidir, crecimiento } \\
\text { cualitativo, desarrollo con equi- } \\
\text { dad y prudencia ecológica. }\end{array}$ & $\begin{array}{l}\text { Biofísico (Ecológico), } \\
\text { Institucional, Económi- } \\
\text { co, Social. }\end{array}$ \\
\hline
\end{tabular}




\begin{tabular}{|c|c|c|c|}
\hline Paradigma & Concepto de desarrollo & Planteamiento & Dimensiones \\
\hline $\begin{array}{l}\text { Administración } \\
\text { de Recursos } \\
\text { (Manejo) } \\
\text { 1970-1980 }\end{array}$ & $\begin{array}{l}\text { Crecimiento económico aso- } \\
\text { ciado al stock de recursos } \\
\text { naturales (valores crematís- } \\
\text { ticos), preocupación por el } \\
\text { sector secundario, enfoque } \\
\text { de mercados. Inclusión de } \\
\text { los recursos naturales en los } \\
\text { balances nacionales. }\end{array}$ & $\begin{array}{l}\text { Antropocéntrico, globalizar la } \\
\text { preocupación existente sobre los } \\
\text { activos naturales y su incidencia } \\
\text { en el desarrollo de los países }\end{array}$ & $\begin{array}{l}\text { Biofísica (Materiales y } \\
\text { entorno), Económico (In- } \\
\text { dustria), Institucional. }\end{array}$ \\
\hline $\begin{array}{l}\text { Desarrollo Sos- } \\
\text { tenible (DS) } \\
1980-1990\end{array}$ & $\begin{array}{l}\text { El desarrollo que satisface } \\
\text { las necesidades del presente } \\
\text { sin comprometer la habili- } \\
\text { dad de las generaciones fu- } \\
\text { turas de satisfacer sus propias } \\
\text { necesidades. }\end{array}$ & $\begin{array}{l}\text { Proceso de cambio (mejoramien- } \\
\text { to) que se puede mantener en } \\
\text { el tiempo (Sostenibilidad como } \\
\text { principio funcional), los obje- } \\
\text { tivos que persiga el desarrollo } \\
\text { determinaran si este es soste- } \\
\text { nible o no. }\end{array}$ & $\begin{array}{l}\text { Biofísico (Ecológico), } \\
\text { Económico, Social. }\end{array}$ \\
\hline $\begin{array}{l}\text { Posdesarrollo } \\
1980-1990\end{array}$ & $\begin{array}{l}\text { Definición (?) del desarrollo } \\
\text { en términos de Buen Vivir } \\
\text { (bienestar integral y colec- } \\
\text { tivo). Opuesto al discurso de } \\
\text { Desarrollo hegemónico de } \\
\text { los países industrializados } \\
\text { (Crecimiento económico). }\end{array}$ & $\begin{array}{l}\text { Crítica posestructuralista, cons- } \\
\text { trucción de alternativas al desa- } \\
\text { rrollo (No alternativas de desa- } \\
\text { rrollo). Estudios pluriversales, } \\
\text { visión de la Tierra como un todo } \\
\text { viviente siempre emergente. }\end{array}$ & $\begin{array}{l}\text { Social, Cultural, Bio- } \\
\text { físico, Institucional, } \\
\text { Económico. }\end{array}$ \\
\hline $\begin{array}{l}\text { Gestión de los } \\
\text { Ecosistemas } \\
1980-1990\end{array}$ & $\begin{array}{l}\text { En la línea del Desarrollo } \\
\text { Sostenible. }\end{array}$ & $\begin{array}{l}\text { Cientificista, gestión multisecto- } \\
\text { rial e interdisciplinaria soporta- } \\
\text { da en el conocimiento ajustable } \\
\text { a nuevos retos mediante el mo- } \\
\text { nitoreo e investigación. }\end{array}$ & $\begin{array}{l}\text { Institucional, Social (Co- } \\
\text { nocimiento), Biofísica. }\end{array}$ \\
\hline $\begin{array}{l}\text { Gestión } \\
\text { Ambiental } \\
\text { Integrada } \\
1990\end{array}$ & $\begin{array}{l}\text { Crecimiento económico y } \\
\text { maximización de la ganan- } \\
\text { cia asociada a un mane- } \\
\text { jo adecuado de residuos y } \\
\text { contaminantes }\end{array}$ & $\begin{array}{l}\text { Sectorial, preventivo de mejo- } \\
\text { ra continua, ciclos de energía y } \\
\text { materiales. }\end{array}$ & $\begin{array}{l}\text { Económico (Industria), } \\
\text { Biofísica (Materiales y } \\
\text { entorno), Institucional. }\end{array}$ \\
\hline $\begin{array}{l}\text { Gestión Adapta- } \\
\text { tiva (Cogestión) } \\
(1986)-1990\end{array}$ & $\begin{array}{l}\text { Desarrollo como proceso di- } \\
\text { námico ajustable basado en la } \\
\text { reducción de la incertidumbre } \\
\text { a través del monitoreo y el } \\
\text { aprendizaje. }\end{array}$ & $\begin{array}{l}\text { Adaptativo, principio de precau- } \\
\text { ción, monitoreo, seguimiento } \\
\text { riguroso y ajuste constante. }\end{array}$ & $\begin{array}{l}\text { Social (Conocimiento), } \\
\text { Biofísica, Institucional y } \\
\text { Económica. }\end{array}$ \\
\hline $\begin{array}{l}\text { Ecoinnovación } \\
1990\end{array}$ & $\begin{array}{l}\text { Asociado al Mercado, rela- } \\
\text { cionado con nuevos produc- } \\
\text { tos y procesos que agregan } \\
\text { valor pero disminuyen sig- } \\
\text { nificativamente el impacto } \\
\text { ambiental. }\end{array}$ & $\begin{array}{l}\text { Enfoque positivista de optimis- } \\
\text { mo tecnológico - Interacción } \\
\text { balanceada entre economía, } \\
\text { conocimiento (tecnologías) y } \\
\text { ambiente. }\end{array}$ & $\begin{array}{l}\text { Económica, Social (CTeI), } \\
\text { Biofísica. }\end{array}$ \\
\hline
\end{tabular}




\begin{tabular}{|c|c|c|c|}
\hline Paradigma & Concepto de desarrollo & Planteamiento & Dimensiones \\
\hline $\begin{array}{l}\text { Decrecimiento } \\
\text { sostenible } \\
1990-2000\end{array}$ & $\begin{array}{l}\text { Economías alternativas sus- } \\
\text { tentadas en una profunda } \\
\text { comprensión y preocupación } \\
\text { acerca de los límites físicos y } \\
\text { sociales existentes (Opuesto } \\
\text { al crecimiento). Incorpora la } \\
\text { Justicia ambiental. }\end{array}$ & $\begin{array}{l}\text { Termodinámico, la economía } \\
\text { debe decrecer físicamente en } \\
\text { términos de los Flujos de Mate- } \\
\text { riales, la Energía, la HANPP y } \\
\text { del uso de agua. }\end{array}$ & $\begin{array}{l}\text { Biofísica, Social (Justi- } \\
\text { cia), Cultural, Institucio- } \\
\text { nal y Económica. }\end{array}$ \\
\hline
\end{tabular}

Fuente: elaboración propia

Si bien los paradigmas presentan diferentes tipos de relaciones entre sus planteamientos, como se ilustra en la tabla 1, es posible afirmar que comparten las siguientes limitaciones:

i. Carecen de una dimensión glocal ambiental.

ii. No abordan las relaciones multidimensionales existentes en entornos particulares propios de los sistemas heterogéneos.

iii. No logran trascender el modelo conceptual de desarrollo vía crecimiento económico o solución técnica asociados al esquema de mercado tradicional sin avanzar hacia las posibilidades que generan las sociedades del conocimiento.

iv. En este sentido, invisibilizan la existencia de otros imaginarios de desarrollo que deben compatibilizarse en múltiples escalas.

v. No incorporan el modo II de hacer ciencia, donde los saberes son fundamentales en la dirección para el desarrollo integral en entornos glocales.

vi. Requieren un mayor esfuerzo conceptual en la construcción de propósitos comunes de tipo ambiental, acordados entre actores que representan grupos sociales y sectores particulares, para posicionar los imaginarios culturales propios y evitar los reduccionismos epistémicos.

Considerando estos elementos, los nuevos paradigmas de la gestión ambiental deben orientarse hacia la construcción de conceptos que relacionen de forma integral la oferta ecológica, las lógicas productivas, los actores del territorio que intervienen sobre el entorno y los propósitos regionales/locales en un contexto globalizado para el desarrollo sostenible.

\section{LITERATURA CITADA}

[1] G. Gallopín, Sostenibilidad y desarrollo sostenible: un enfoque sistémico, Santiago de Chile 2003, 46 p. 
[2] F. Monica, "Sustainable Development - a Territorial Development Premise of Galaţi," Procedia-Social and Behavioral Sciences, vol. 46, n. ${ }^{\circ}$ 0, pp. 1506-1509, //, 2012.

[3] F. Fahy, y M. Ó Cinnéide, "Developing and testing an operational framework for assessing quality of life," Environmental Impact Assessment Review, vol. 28, n. ㅇ 6, pp. 366-379, 8//, 2008.

[4] E. F. Lambin, y P. Meyfroidt, "Global land use change, economic globalization, and the looming land scarcity," Proceedings of the National Academy of Sciences, vol. 108, no. 9, pp. 3465-3472, March 1, 2011, 2011.

[5] UNEP, y WRI, Ecosystems and Human Well-Being : a framework for assessment, Washington D.C.: Island press - World Resources Institute, 2003, 245 p.

[6] J. Antequera et al., "Sostenibilidad y desarrollo sostenible: un modelo por construir," Sostenible?, n. 7 , pp. 98-118, 2005.

[7] B. Martín et al., "The conservation against development paradigm in protected areas: Valuation of ecosystem services in the Doñana social-ecological system (southwestern Spain)," Ecological Economics, vol. 70, n. ${ }^{\circ}$ 8, pp. 1481-1491, 2011.

[8] J. Cary, y A. Roberts, "The limitations of environmental management systems in Australian agriculture," Journal of Environmental Management, vol. 92, n. ${ }^{\circ 3}$, pp. 878-885, 2011.

[9] I. Valentine et al., "Principles and processes for effecting change in environmental management in New Zealand," Journal of Environmental Management, vol. 82, n. ${ }^{\circ}$, pp. 311-318, 2007.

[10] M. Lozano, y J. Vallés, "An analysis of the implementation of an environmental management system in a local public administration," Journal of Environmental Management, vol. 82, n. ${ }^{\circ}$ 4, pp. 495-511, 2007.

[11] T. J. Kallio, y P. Nordberg, "The Evolution of Organizations and Natural Environment Discourse," Organization \& Environment, vol. 19, n. ${ }^{\circ}$ 4, pp. 439-457, December 1, 2006, 2006.

[12] R. Arrué et al., Gestión ambiental a nivel local, Santiago de Chile: Corporación Ambiental del Sur, 2005, 95 p.

[13] D. S. Steingard et al., "Exploring the Frontiers of Environmental Management: A Natural Law-based Perspective," Journal of Human Values, vol. 10, n. ${ }^{\circ}$ 2, pp. 79-97, October 1, 2004, 2004.

[14] M. Rodríguez, y G. Espinoza, Gestión ambiental en América Latina y el Caribe : evolución, tendencias y principales prácticas, p.`pp. 286, Washington, D. C.: Banco Interamericano de Desarrollo, 2002, p.

[15] CEPAL, y PNUMA, La sostenibilidad del desarrollo en América Latina y el Caribe: Desafios y oportunidades, Santiago de Chile: UN, 2002, 241 p.

[16] L. J. Hawcroft, y T. L. Milfont, "The use (and abuse) of the new environmental paradigm scale over the last 30 years: A meta-analysis," Journal of Environmental Psychology, vol. 30, n. ${ }^{\circ}$, pp. 143-158, 2010. 
[17] I. Linkov et al., "From optimization to adaptation: Shifting paradigms in environmental management and their application to remedial decisions," Integrated Environmental Assessment and Management, vol. 2, n. ${ }^{\circ}$ 1, pp. 92-98, 2006.

[18] M. Pérez et al., Desarrollo sostenible: Principios, aplicaciones y lineamientos de política para Colombia, Cali, Colombia. Universidad del Valle-Instituto CINARA, 2010, 346 pp.

[19] A. J. Bebbington, La glocalización de la gobernanza ambiental: relaciones de escala en los movimientos socio ambientales y sus implicaciones para la gobernanza ambiental en zonas de influencia minera en el Perú y el Ecuador, Manchester: Universidad de Manchester-Escuela de Medio Ambiente y Desarrollo, 2006, 55 p.

[20] A. Escobar, "Una ecología de la diferencia: Igualdad y conflicto en un mundo glocalizado", en Más allá del Tercer Mundo. Globalización y Diferencia., pp. 123-144., Bogotá. D. C.: ICANH-Universidad del Cauca., 2005.

[21] M. Colby, The Evolution of Paradigms of Environmental Management in Development, Reporte, World Bank, Washington D. C., 1989.

[22] A. Figueroa et al., Evaluación de impacto ambiental, un instrumento para el desarrollo. En: La evaluación de impacto ambiental (EIA), Cali: Centro de Estudios Ambientales para el Desarrollo Regional, 1998, p.

[23] I. Sachs, "Ecodesarrollo: concepto, aplicación, beneficios y riesgos" Agricultura y Sociedad, vol. 18, pp. 9-32, 1981.

[24] C. R. Negrão, "Gestión Ambiental”, en Notas de Clase II CURSO INTERNACIONAL DE ASPECTOS GEOLÓGICOS DE PROTECCIÓN AMBIENTAL, U. y. UNICAMP, ed., pp. 2735, Campinas, SP, Brasil: Unesco y UNICAMP, 2000.

[25] E. Urteaga, "Las teorías alternativas del desarrollo sostenible," Boletín de la Asociación de Geógrafos Españoles, vol. 55, pp. 113-126, 2011.

[26] G. Bruntland, Nuestro futuro común, Oxford: Naciones Unidas. Oxford University Press., $1987, \mathrm{p}$.

[27] G. Gallopín, "El desarrollo sostenible desde una perspectiva sistémica”, Sostenible, n. ${ }^{\circ}$ 11, pp. 17-35, 2010.

[28] J. Rockström et al., "Sustainable Development and Planetary Boundaries.," presentado en High Level Panel on the Post-2015 Development Agenda 2013, pp. 46.

[29] J. Harlow et al., "A Review of Utopian Themes in Sustainable Development Discourse," Sustainable Development, vol. 21, n. ${ }^{\circ}$ 4, pp. 270-280, 2013.

[30] A. Escobar, "El "posdesarrollo" como concepto y práctica social", en Políticas de economía, ambiente y sociedad en tiempos de globalización., D. Mato, ed., pp. 17-31, Caracas: Facultad de Ciencias Económicas y Sociales, Universidad Central de Venezuela, 2005.

[31] A. Escobar, "Más allá del desarrollo: posdesarrollo y transiciones hacia el pluriverso," Revista de Antropología Social, vol. 21, pp. 23-62, 2011. 
[32] M. Mandujano, "Postdesarrollo, Modernidad y otros mundos; Entrevista con Arturo Escobar," Oxímora Revista Internacional de Ética y Política, vol. 2, pp. 233-248, 2013.

[33] C. Bennett, "Supporting the Posts in Development Discourse: Under-development, Overdevelopment, Post-development," Sociology Compass, vol. 6, n. ํ 12, pp. 974-986, 2012.

[34] N. L. Christensen et al., "The Report of the Ecological Society of America Committee on the Scientific Basis for Ecosystem Management”, Ecological Applications, vol. 6, n. ${ }^{\circ}$, pp. 665-691, 1996.

[35] S. Born, y W. Sonzogni, "Integrated environmental management: strengthening the conceptualization”, Environmental Management, vol. 19, n. 2, pp. 167-181, 1995/03/01, 1995.

[36] D. Nawrocka, y T. Parker, "Finding the connection: environmental management systems and environmental performance", Journal of Cleaner Production, vol. 17, n. ${ }^{\circ}$ 6, pp. 601-607, 4//, 2009.

[37] A. Zorpas, "Environmental management systems as sustainable tools in the way of life for the SMEs and VSMEs", Bioresource Technology, vol. 101, n. ${ }^{\circ}$ 6, pp. 1544-1557, 3//, 2010.

[38] D. G. Hole et al., "Manejo adaptativo para la conservación de la biodiversidad frente al cambio climático, perspectiva en los Andes tropicales", en Cambio climático y biodiversidad en los Andes tropicales, S. Herzog et al., eds., pp. 23-55, Paris: IAA, 2012.

[39] C. R. Allen et al., "Adaptive management for a turbulent future", Journal of Environmental Management, vol. 92, n. ${ }^{\circ}$ 5, pp. 1339-1345, 2011.

[40] H. R. Heinimann, "A concept in adaptive ecosystem management-An engineering perspective", Forest Ecology and Management n. ${ }^{\circ} 259$, pp. 848-856, 2010.

[41] F. Berkes, "Evolution of co-management: Role of knowledge generation, bridging organizations and social learning", Journal of Environmental Management, vol. 90, n. ${ }^{\circ}$ 5, pp. 1692-1702, 4//, 2009.

[42] D. Armitage et al., "Adaptive co-management and the paradox of learning", Global Environmental Change, vol. 18, n. ${ }^{\circ}$ 1, pp. 86-98, 2//, 2008.

[43] P. del Río et al., "Enfoques y políticas de eco-innovación. Una visión crítica”, Ekonomiaz: Revista Vasca de Economía, n. ${ }^{\circ}$ 75, pp. 84-111, 2010.

[44] J. Carrillo et al., "Diversity of eco-innovations: Reflections from selected case studies", Journal of Cleaner Production, vol. 18, n. ${ }^{\circ}$ 10-11, pp. 1073-1083, 7//, 2010.

[45] E. Karakaya et al., "Diffusion of eco-innovations: A review", Renewable and Sustainable Energy Reviews, vol. 33, n. ${ }^{\circ}$, pp. 392-399, 5//, 2014.

[46] J. Nill, y R. Kemp, "Evolutionary approaches for sustainable innovation policies: From niche to paradigm?", Research Policy, vol. 38, n. ${ }^{\circ}$ 4, pp. 668-680, 5//, 2009.

[47] N. Hofstra, y D. Huisingh, "Eco-innovations characterized: a taxonomic classification of relationships between humans and nature", Journal of Cleaner Production, vol. 66, n. ${ }^{\circ}$, pp. 459-468, 3/1/, 2014. 
[48] F. Schneider et al., "Crisis or opportunity? Economic degrowth for social equity and ecological sustainability. Introduction to this special issue", Journal of Cleaner Production, vol. 18, n. ${ }^{\circ}$, pp. 511-518, 2010.

[49] J. Martínez Alier, “Decrecimiento sostenible: París, abril del 2008,” [En línea], Disponible, 2008.

[50] F. Sekulova et al., "Degrowth: from theory to practice", Journal of Cleaner Production, vol. 38, n. ${ }^{\circ}$ 0, pp. 1-6, 1//, 2013. 
